\title{
Analysis and Modelling of Muscles Motion during Whole Body Vibration
}

\author{
M. Cesarelli, ${ }^{1}$ A. Fratini, ${ }^{1}$ P. Bifulco, ${ }^{1}$ A. La Gatta, ${ }^{2}$ M. Romano, ${ }^{1}$ and G. Pasquariello ${ }^{1}$ \\ ${ }^{1}$ Department of Biomedical Electronic and Telecommunication Engineering, University of Naples "Federico II", 80125 Naples, Italy \\ ${ }^{2}$ Math4Tech Centre, University of Ferrara, Ferrara, 44100 Ferrara, Italy
}

Correspondence should be addressed to M. Cesarelli, cesarell@unina.it

Received 1 May 2009; Accepted 3 August 2009

Academic Editor: João Manuel R. S. Tavares

Copyright ( $) 2010$ M. Cesarelli et al. This is an open access article distributed under the Creative Commons Attribution License, which permits unrestricted use, distribution, and reproduction in any medium, provided the original work is properly cited.

\begin{abstract}
The aim of the study is to characterize the local muscles motion in individuals undergoing whole body mechanical stimulation. In this study we aim also to evaluate how subject positioning modifies vibration dumping, altering local mechanical stimulus. Vibrations were delivered to subjects by the use of a vibrating platform, while stimulation frequency was increased linearly from 15 to $60 \mathrm{~Hz}$. Two different subject postures were here analysed. Platform and muscles motion were monitored using tiny MEMS accelerometers; a contra lateral analysis was also presented. Muscle motion analysis revealed typical displacement trajectories: motion components were found not to be purely sinusoidal neither in phase to each other. Results also revealed a mechanical resonant-like behaviour at some muscles, similar to a second-order system response. Resonance frequencies and dumping factors depended on subject and his positioning. Proper mechanical stimulation can maximize muscle spindle solicitation, which may produce a more effective muscle activation.
\end{abstract}

\section{Introduction}

Vibratory stimulations transferred to the whole body, that is, whole body vibrations (WBVs) treatments, have recently arouse a great deal of interest. WBVs are widely studied in the fields of exercise physiology, sport, and rehabilitation medicine [1-4]. Many studies have indeed accounted for their impact on metabolic and hormonal activity, neuromuscular response, and postural control [5-21].

WBV training aims to mechanically activate muscles by eliciting reflex neuromuscular activity. In such treatments, vibrations are generally delivered to the body as a whole by the use of vibrating plates, while muscular activity estimation is assessed analysing the simultaneous electromyography (EMG) activity, in particular the EMGs root mean square (RMS)values.

These vibrating devices produce vertical sinusoidal oscillations (or alternating rotation) of a platform across a frequency range from 10 to $80 \mathrm{~Hz}$ and peak-to-peak displacements from 1 to $10 \mathrm{~mm}[3,22]$.

Mostly, individuals undergoing WBV treatments are requested to hold a specific position onto the plate. Vibratory stimulation, impressed by the platform, transfers to the muscles through the body.

When vibration loads are applied, mechanical and metabolic responses arise from the neuromuscular system [2, 7, 9, 17]; muscles increase their activity to dampen the soft tissue oscillations that occur in response to vibrations Specific WBV frequencies also seem to produce a higher EMGs RMS values than others [2].

Although EMG analysis is a common practice in estimating muscle response to vibratory stimulations, EMG is difficult to measure because vibrations induced in the tissues causes movement artifacts, which interfere with measurement of muscle activity $[23,24]$.

However, as reported in a previous study, there is a significant correlation between muscle motion and EMG activity [25]. Most of vibration-induced neuromuscular response occurs at the resonant frequencies of the tissues, concurring with the highest levels of muscle activity [23, 26-28].

Local mechanical stimulus characterization (i.e., muscle motion analysis) could be meaningful in discovering proper muscle stimulation and may contribute to suggest appropriate and effective WBV exercise protocols. 


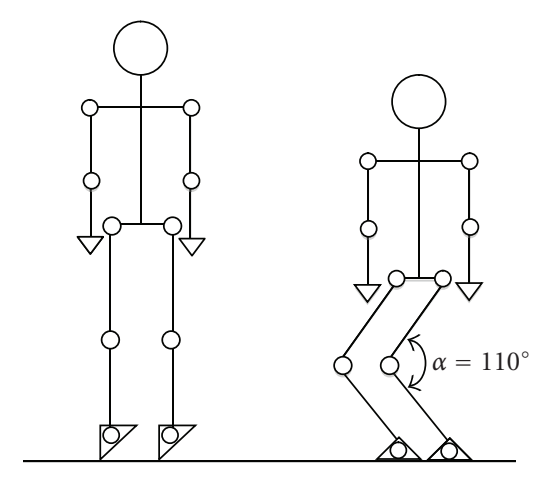

Figure 1: Body postures selected for the study.

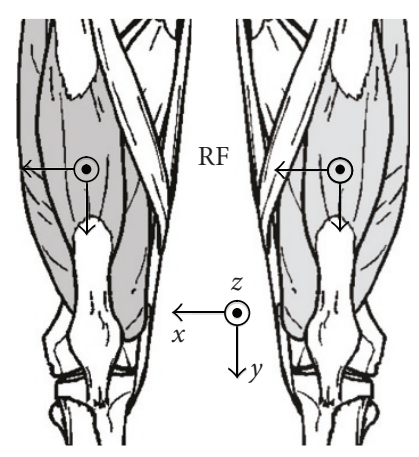

Figure 2: Example of accelerometer arrangement on muscles for both of the lower limbs. The picture shows the position and orientation of the accelerometer on RF muscles.

WBV effects depend on intensity, direction and frequency of vibratory stimulation; however, subject training posture should be considered as one of the most important factors involved.

This study concentrates on the characterization and modelling of the muscle motion in individuals exposed to whole body mechanical stimulation while holding different postures on platform. In our study we aim also to evaluate how subject positioning on the platform modifies vibration dumping through the body and in turn, the local vibratory stimulus. However, only two postures were analysed: hack squat position and upright position with heel raised.

Patients were impressed by a linear vibratory stimulation sweep in a frequency range from 15 to $60 \mathrm{~Hz}$, while motions of the Rectus Femoris (RF), Biceps Femoris (BF)- (long head) and Gastrocnemius Lateralis (GL) were analyzed; a contralateral analysis between both of the lower limbs was also presented.

Muscle motion analysis showed typical 3D trajectories. Muscle displacement was found of different amount on each relative axis; motion components were also found not to be purely sinusoidal neither in phase to each other.

Results revealed a mechanical resonant-like behaviour at some muscles, very similar to a second-order system in the frequency range explored. A simple data fitting was then performed and it confirmed a high correlation $\left(R_{1}^{2}\right)$ between model and experimental data.

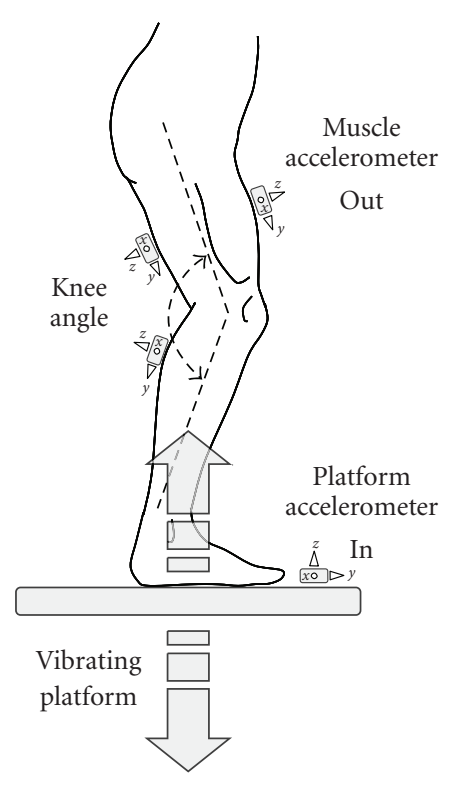

Figure 3: Scheme of measurement setup.

Contralateral analysis showed that the mechanical behaviour of muscles (both dominant and non dominant leg) was similar. Two limbs muscles motion resulted to be synchronous and symmetric with respect to the sagittalplane.

\section{Methods}

2.1. Subjects, Device, and Vibratory Stimulus. Twenty healthy males (age $22.6 \pm 2.2$ years, height $176.7 \pm 8.4 \mathrm{~cm}$, weight $74.0 \pm 10.1 \mathrm{~kg}$-athletically untrained), not affected by any known neurological or musculoskeletal disorder, were voluntarily involved in the study and gave their informed, written consent to participate. Vibrations were impressed by using a vibrating platform (TSEM S.p.A., Padova-Italy). Platform displacement was exclusively vertical, sinusoidal with an intensity (peak-to-peak displacement) set to $1.2 \mathrm{~mm}$. The plate frequency was controlled from a function generator (Krohn-Hite 5300A) to obtain a linear vibratory stimulation sweep in a frequency range from 15 to $60 \mathrm{~Hz}$. Frequency sweep time interval was set to 26 seconds, corresponding to an increase rate of about $1.7 \mathrm{~Hz}$ per second. All the subject were familiarized with the device and proper positioning before recording; during the test subjects were requested to hold two positions (see Figure 1) without the use of any constraint:

(i) P1: hack squat position: in the hack squat posture, subjects bent their legs at the knees with an angle of $110^{\circ}$ between the lower legs and the upper legs;

(ii) P2: upright on feet toes: in upright posture, subjects stand on their feet toes with heel raised.

These postures were selected as they are two of the manufacturers suggested positions for WBV training; they also reduce transmission of vibrations to patient's trunk and head [29]. 


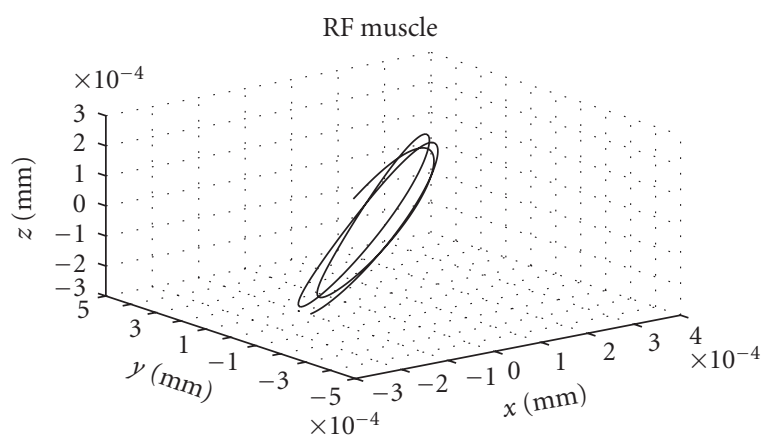

(a)

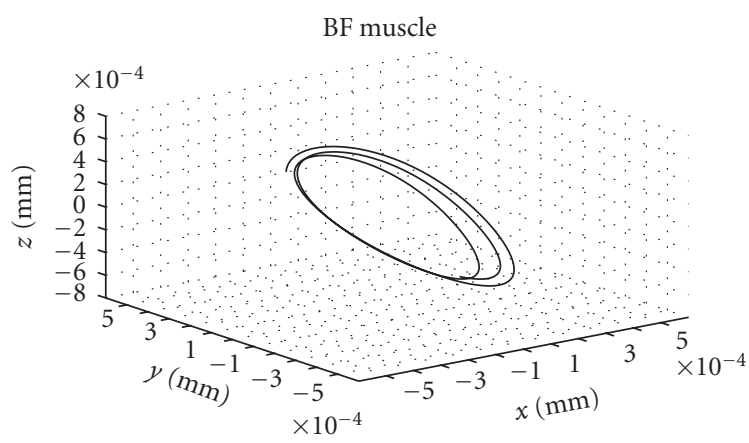

(b)

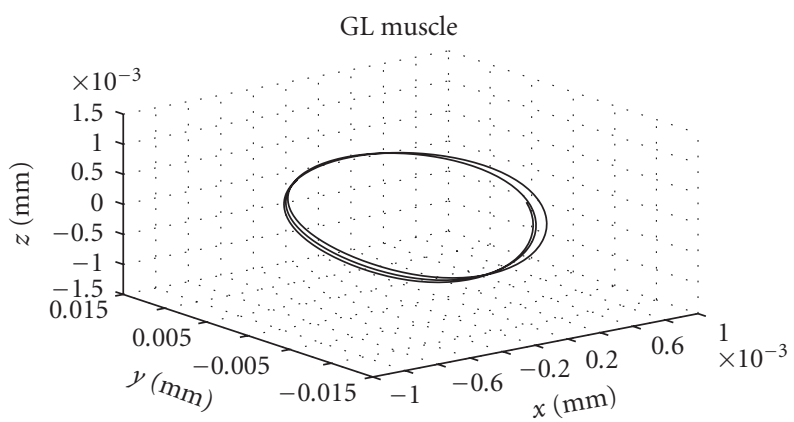

(c)

Figure 4: An example of three dimensional displacement of RF, BF and GL muscle belly under vibratory stimulation. The subject was holding the hack squat position while the frequency of the vibratory stimulus was around to $24 \mathrm{~Hz}$. The figure depicts few cycles. The reference position for each muscle was placed at the accelerometer axis'origin.

Tiny and lightweight (less than $10 \mathrm{~g}$ ) three-axial MEMS accelerometers (Freescale Semiconductors) were used to measure accelerations onto patient's skin and on the vibrating platform (the sensors were set to measure acceleration within a $\pm 10 \mathrm{~g}$ range).

The accelerometers were placed at $50 \%$ on the line from the anterior spina iliaca superior to the superior part of the patella for RF muscle, at $50 \%$ on the line between the ischial tuberosity and the lateral epicondyle of the tibia for BF muscle, at $1 / 3$ of the line between the head of the fibula and the heel for GL muscle and at the centre of the vibration plate to monitor mechanical vibration (Figure 3). MEMS accelerometers were stuck onto muscles belly with medical adhesive tape (3 M). For each postural position, signals from the muscle bellies (both dominant and nondominant leg) were collected contemporaneously (Figure 2).

A PC multi-channel 16-bit data acquisition card (NIDAQCard 6251) was used to acquire acceleration signals. All signals were sampled at $2048 \mathrm{~Hz}$.

As mentioned, each of the acquisition (one for each posture), spaced with 60 seconds rest intervals, had a duration of 26 seconds; during this time interval the stimulation frequency, imposed by vibrating plate, raises linearly from 15 to $60 \mathrm{~Hz}$.

2.2. Accelerometer Data Processing. Accelerations signals provided information related to muscle belly oscillation; they were preprocessed in order to exclude influence of gravity and slow accommodation movements on motion information. Since gravity and subject's accommodation movements contributions are typically confined from 0 to $5 \mathrm{~Hz}$ [30], recordings have been high-pass filtered using a zero-phase 5th-order Butterworth with a cut off frequency of $10 \mathrm{~Hz}$.

Muscle and platform displacements were estimated integrating twice the acceleration data after high-pass filtering on each axis. The resultant vectors were then estimated and analysed.

2.3. Frequency Response of the Biomechanical System. The frequency response of the biomechanical system was obtained processing the resultant displacement signals. The actual displacement of the vibrating plate (which was substantially constant in amplitude) was considered as the input signal, while the muscle displacement as system output.

Instantaneous frequency was computed for each vibration cycle by estimating two subsequent zero-crossings (with a positive slope) of the signal. Input displacement amplitude, output displacement amplitude and input/output phase difference were estimated for each cycle; gains were obtained by the input/output amplitude ratio. System gain and phase were then plotted against frequency (experimental system response).

2.4. System Identification and Data Fitting. A simple data fitting was performed supposing the system as a pure 

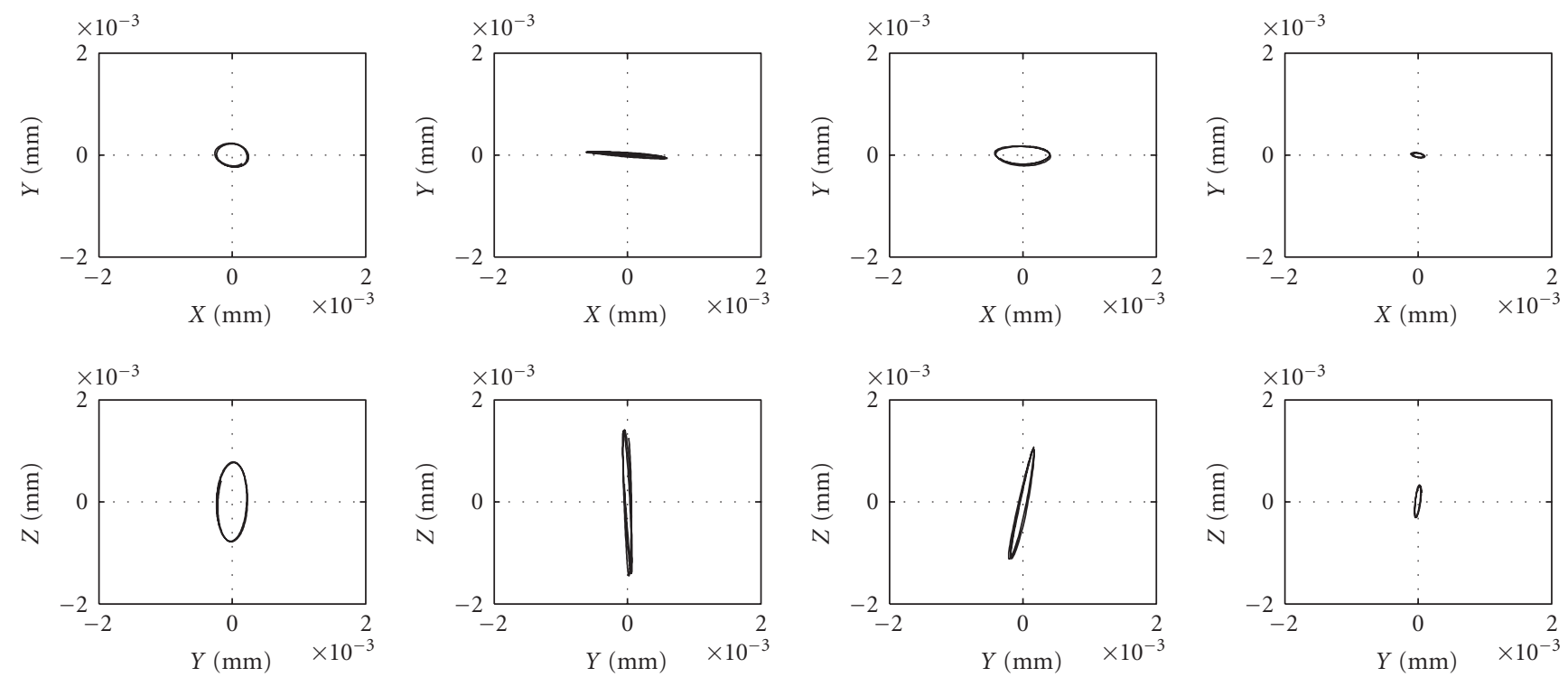

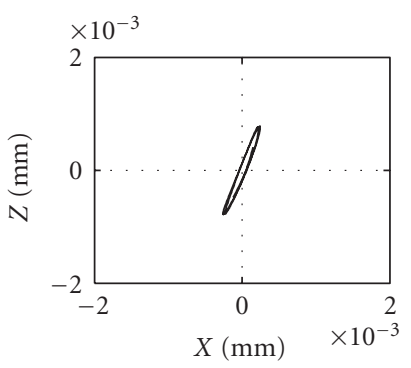

(a)

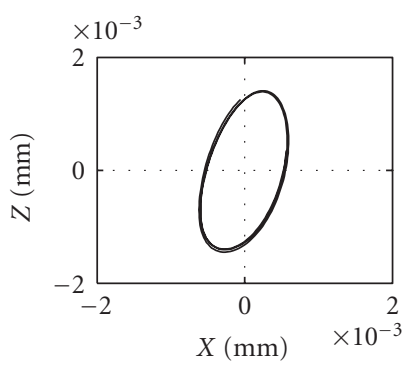

(b)

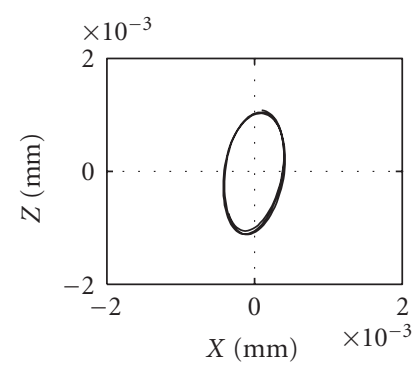

(c)

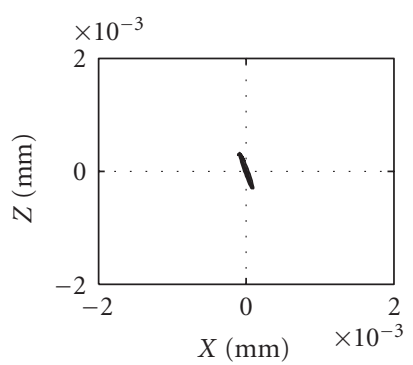

(d)

FIGURE 5: An example of the RF muscle belly trajectories over the $(x, y),(y, z)$ and $(x, z)$ planes, at different stimulation frequencies $((a)=$ $16 \mathrm{~Hz},(\mathrm{~b})=25 \mathrm{~Hz},(\mathrm{c})=40 \mathrm{~Hz},(\mathrm{~d})=50 \mathrm{~Hz})$. The figure corresponds to $\mathrm{P} 1$ subject positioning.

second-order given by the following equation:

$$
G(s)=K_{\mathrm{dc}} \frac{1}{\left(s / \omega_{n}\right)^{2}+2 \xi\left(s / \omega_{n}\right)+1},
$$

where $K_{\mathrm{dc}}$ is the DC gain, $\omega_{n}$ is the natural frequency, and $\xi$ is the dumping ratio.

Model parameters $\left(\omega_{n}\right.$ and $\xi$ ) were estimated by utilizing the values of the dominant peak (amplitude and frequency) of the experimental frequency response; $K_{\mathrm{dc}}$ coefficient was estimated minimizing the root mean squared error (RMSE) between model and experimental data.

2.5. Contralateral Analysis. A contralateral analysis on both of the lower limbs was also conduced by comparing the following:

(i) muscle motion of dominant and nondominant leg,

(ii) the estimated resonance frequencies.

In particular we evaluate the symmetry of muscle motion and the similarity of frequency response between lower limbs. For each posture and for all of the muscles analyzed we computed the correlation coefficient $\left(R_{2}^{2}\right)$ between the estimated frequency responses (amplitude) of the two limbs muscles.

\section{Results}

Muscle motion analysis showed peculiar three-dimensional trajectories. Muscle displacement components resulted different on each relative axis; they were also found not to be purely sinusoidal neither in phase to each other. In Figure 4 are shown typical 3D estimated muscle trajectory over time (few vibration cycles are represented).

Figures 5, 6 and 7 show some more details about the motion of the three analysed muscles over the frequency range explored The image shows the projections of the trajectory over the three planes: $X / Y, Y / Z$ and $X / Z$, at different stimulation frequencies;

In general, acceleration components registered on muscle belly were not in phase one to another. They were also different in amplitude and showed a dissimilar behaviour with the frequency. In general the recorded accelerations confirmed a frequency-dependent increase.

From Figure 5 it is easy to identify a different mechanical response for each axis and, in turn for each plane. Displacements on $x$-, $y$ - and $z$-axis resulted not always in phase, neither pure sinusoids (the figures were not exact ellipses).

From figures it is also noticeable that in position P1, $\mathrm{RF}$ and $\mathrm{BF}$ muscles movements were more pronounced on 

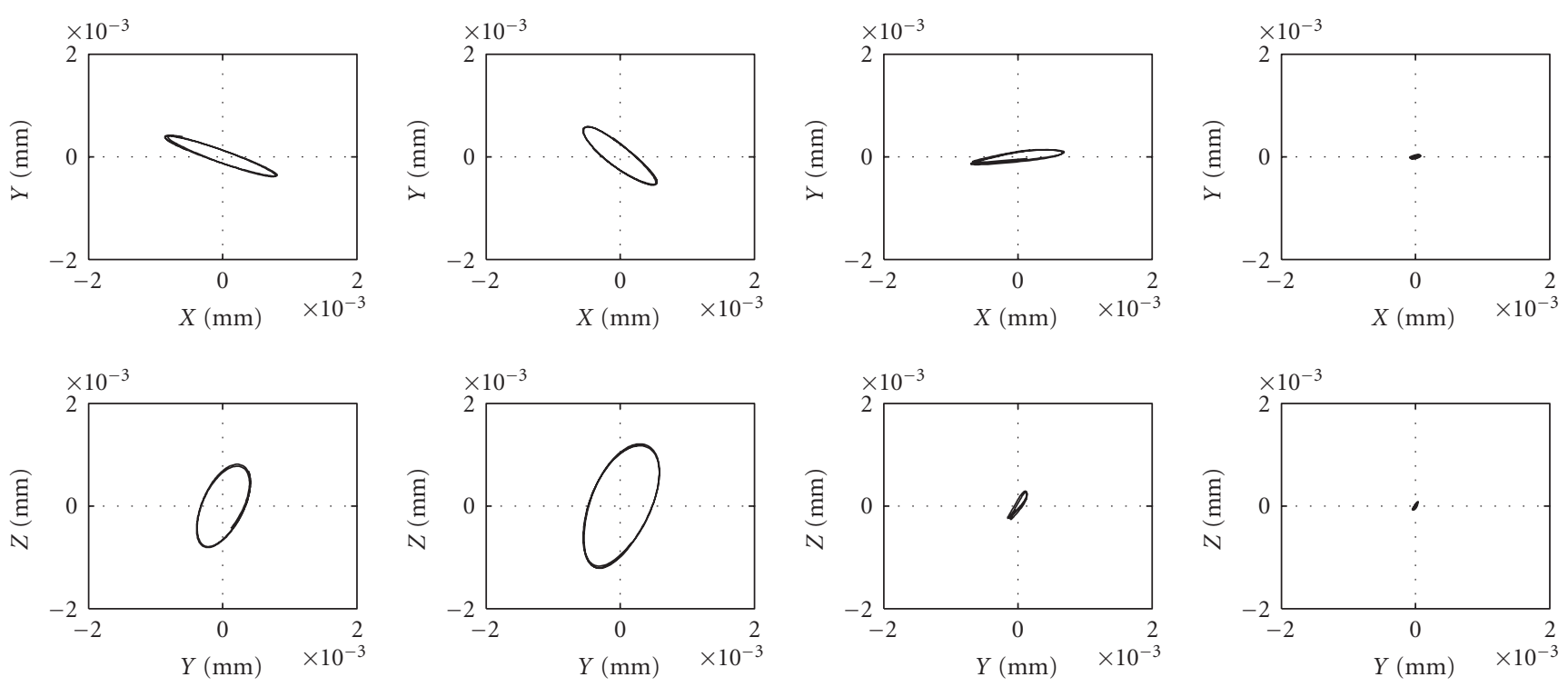

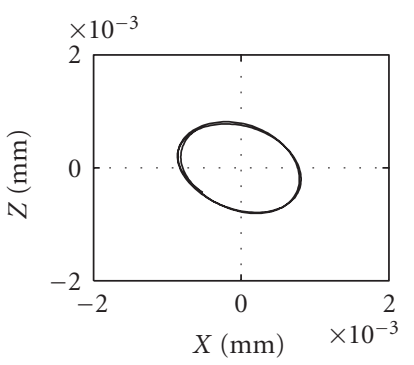

(a)

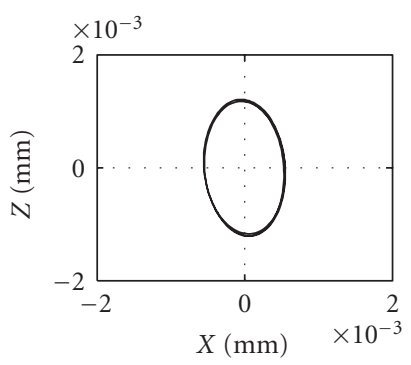

(b)

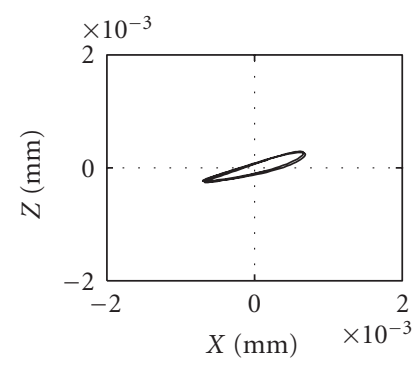

(c)

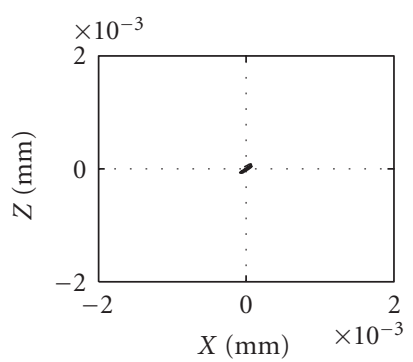

(d)

FIgure 6: An example of the BF muscle belly trajectories over the $(x, y),(y, z)$ and $(x, z)$ planes, at different stimulation frequencies $(($ a) $=$ $16 \mathrm{~Hz},(\mathrm{~b})=25 \mathrm{~Hz},(\mathrm{c})=40 \mathrm{~Hz},(\mathrm{~d})=50 \mathrm{~Hz})$. The figure corresponds to $\mathrm{P} 1$ subject positioning.

$z$-axis; the same result was found in all the subjects (see Figures 5 and 6 ).

In Figure 7 it is shown that in position P1 for GL muscle movements seemed to be more pronounced on $y$ axis.

It is worth mentioning that resonant frequency and dumping factor were subject dependant.

In $\mathrm{P} 1$ position $\mathrm{RF}$ and $\mathrm{BF}$ resultant displacement showed a resonance-like profile over frequency; this was not evident for GL. In P2 position only the GL muscle showed a resonant-like profile while $\mathrm{RF}$ and $\mathrm{BF}$ displacements resulted highly dumped (mean normalized displacement $\leq 0.4(\mathrm{~mm}) /(\mathrm{mm}))$. RF, BF and GL resonance frequencies and dumping factors depended on subject.

In Figure 9 is presented an example of the mechanical behaviour of the three examined muscles, over the frequency range of interest, for one subject and with respect to each posture.

For each posture described, the mechanical response of muscles showing a resonant-like profile was very similar to that of second-order system (Figure 10), as confirmed by the correlation coefficient $R_{1}^{2}$ (Table 1).

In general, the contralateral analysis showed that the mechanical behaviour of muscles was similar.
In P1, mean difference between dominant and non dominant limb resonance frequencies $(\Delta \mathrm{Fr})$ was equal to $0.26( \pm 1.85) \mathrm{Hz}$ for $\mathrm{RF}$ and $0.39( \pm 2.79) \mathrm{Hz}$ for $\mathrm{BF}$ (Table 2). In P2, $\Delta \mathrm{Fr}$ for GL was equal to $0.03 \pm 3.23 \mathrm{~Hz}$. $R_{2}^{2}$ coefficient resulted very high, suggesting a similar motion of contralateral muscles (Table 2).

With respect to position $\mathrm{P} 1$, contralateral analysis revealed that motion of both RF muscles (dominant and non dominant leg) was synchronous and symmetric in the sagittal-plane: both RF muscles phase responses were almost identical along $z$ - and $y$-axes (synchronicity), while they showed a difference of $\pi$ rad along $x$-axis (symmetry with respect to sagittal plane). The same behaviour was found for both of BF muscles.

\section{Discussions}

Interaction of human body with applied vertical whole body vibrations strongly depends on subject's characteristics: anatomy, positioning, muscle stiffness and other individual tissue mechanical properties; some authors also consider the possibility that vibratory stimulation impairs the "muscular memory" process responsible for predictive force [30]. 

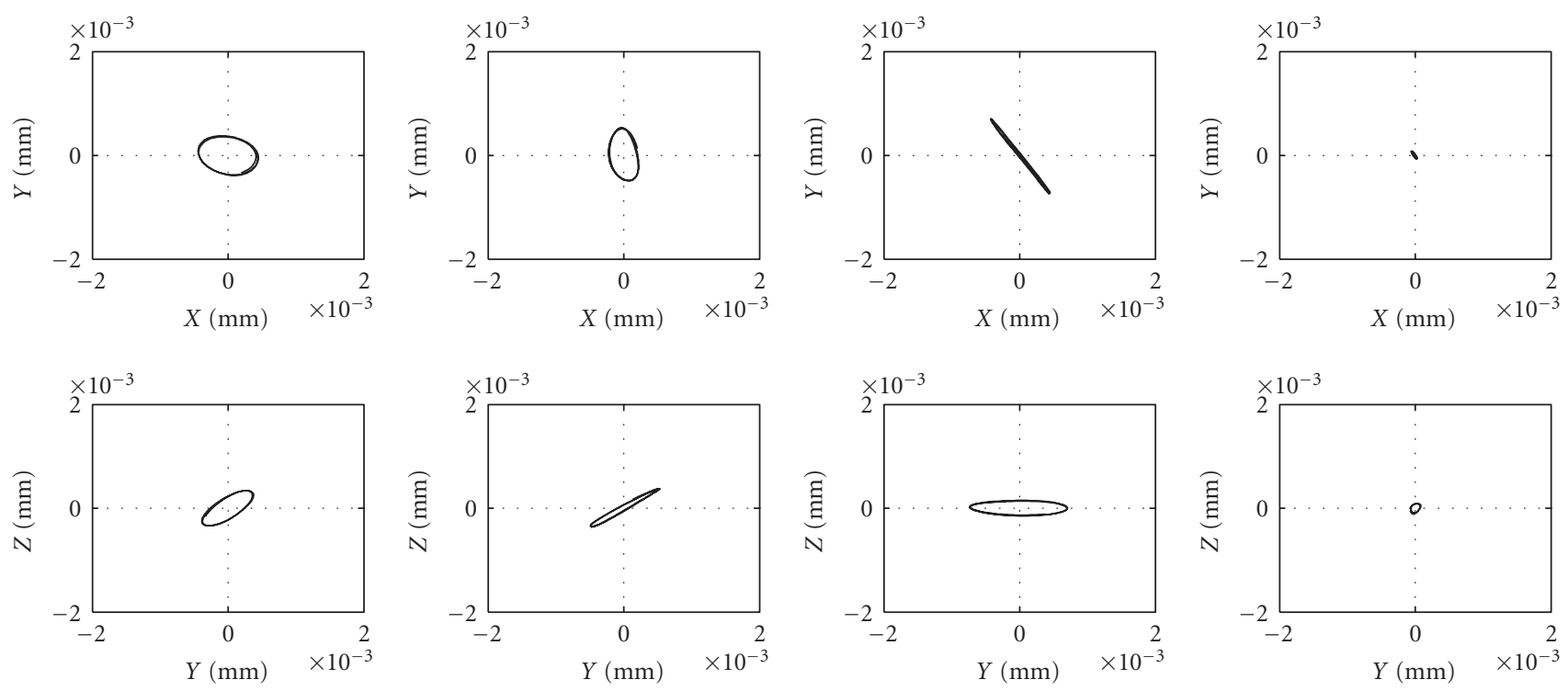

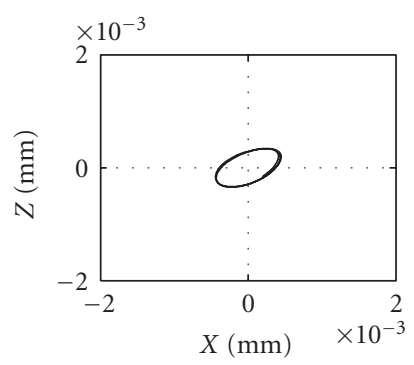

(a)

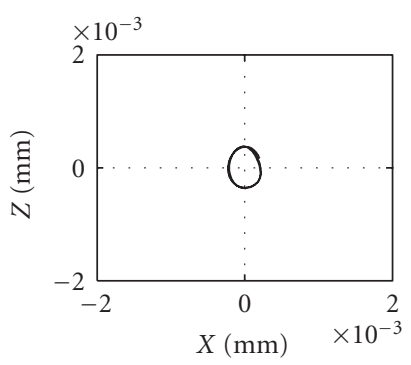

(b)

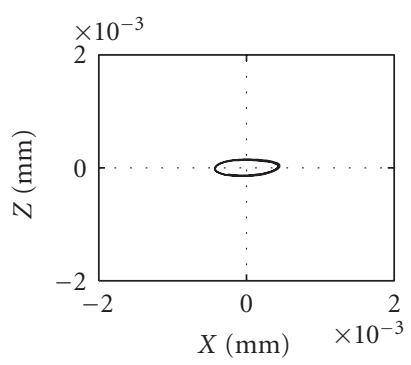

(c)

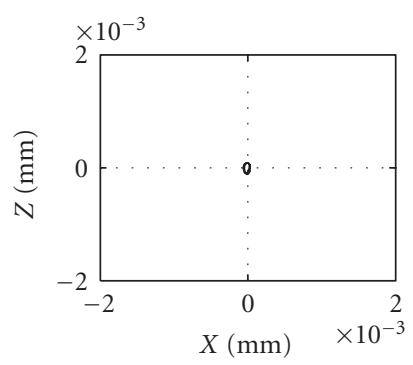

(d)

FIGURE 7: An example of the GL muscle belly trajectories over the $(x, y),(y, z)$ and $(x, z)$ planes, at different stimulation frequencies $((a)=$ $16 \mathrm{~Hz},(\mathrm{~b})=25 \mathrm{~Hz},(\mathrm{c})=40 \mathrm{~Hz},(\mathrm{~d})=50 \mathrm{~Hz})$. The figure corresponds to $\mathrm{P} 2$ subject positioning.

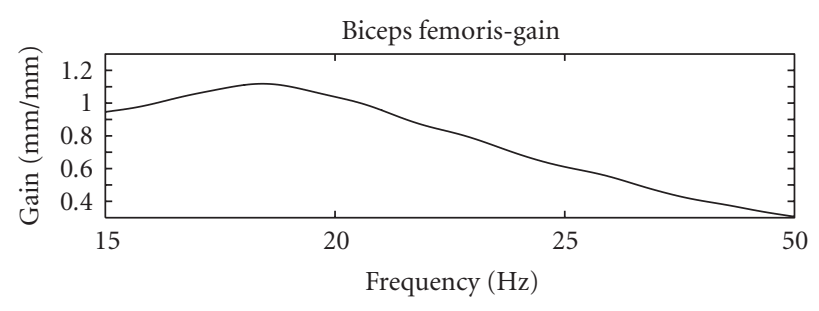

(a)

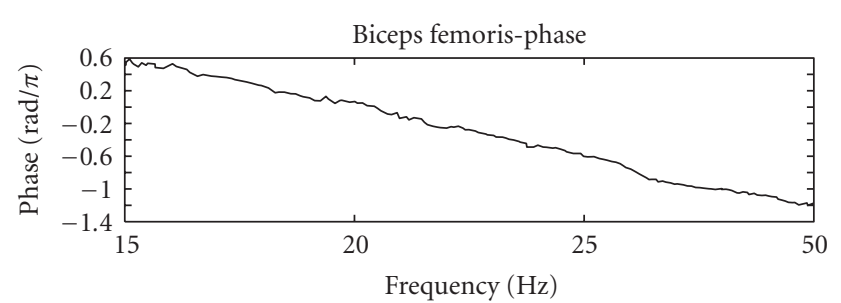

(b)

Figure 8: An example of BF frequency response-(a) amplitude and (b) phase-over frequency with respect to position P1.

Variations of the mentioned parameters modifies the kinematic chain, through which mechanical vibration propagates into the body, altering muscle local mechanical oscillations.

The effects of WBV on muscle motion (i.e., muscle stimulation) depend on intensity, direction and frequency of vibratory stimulation; however, subject training posture, as highlighted by our results, should be considered as one of the most important factors involved.

This study aimed to characterize different lower limb muscle motion in individuals exposed to vertical whole body mechanical stimulation. In our study we analysed how subject positioning on the platform modifies vibration dumping and alters the local vibratory oscillation. Two postures were analysed: hack squat position and upright position with heel raised. Muscle motion was successfully measured using tiny and lightweight MEMS accelerometers.

RF, BF and GL muscles motion was here considered: such kinematics can be expressed as a summation of different terms: muscle mean position (depending exclusively on posture), its vibratory displacements and subjects small accommodation movements.

Those three kinematic components can be easily separated in the frequency domain: the stationary position and the slow, small postural adjustments are confined to the very 


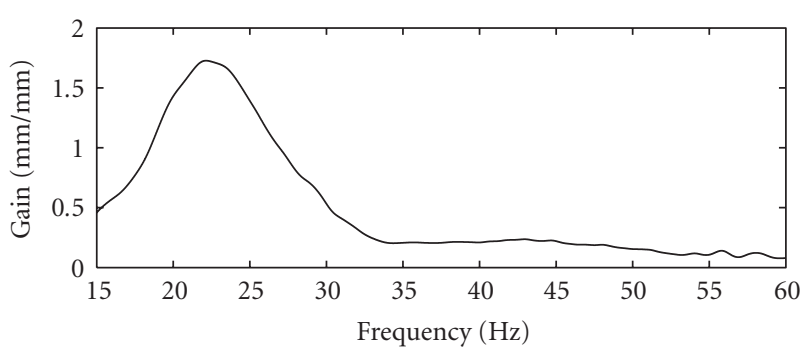

- RF P1

(a)

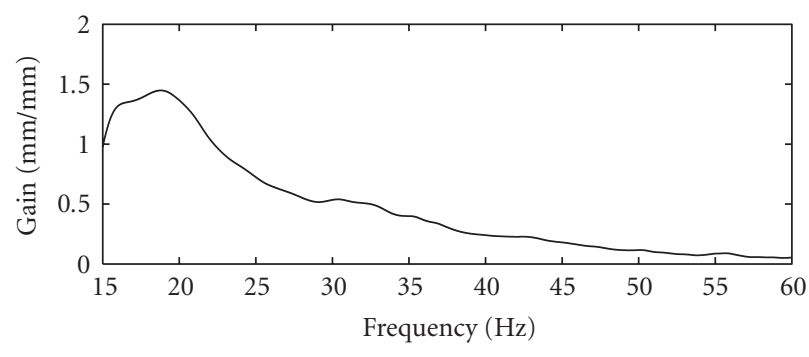

- BF P1

(c)

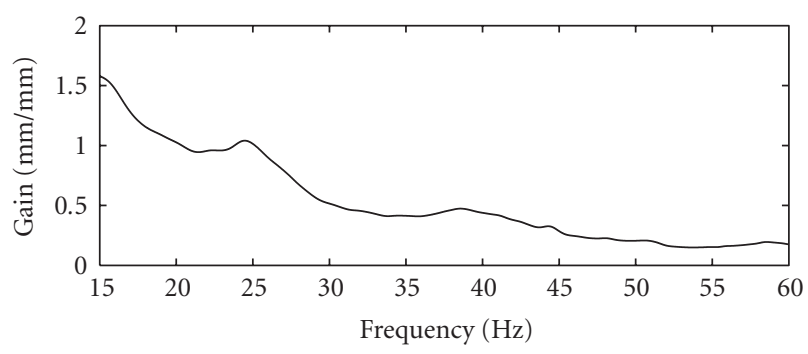

— GL P1

(e)

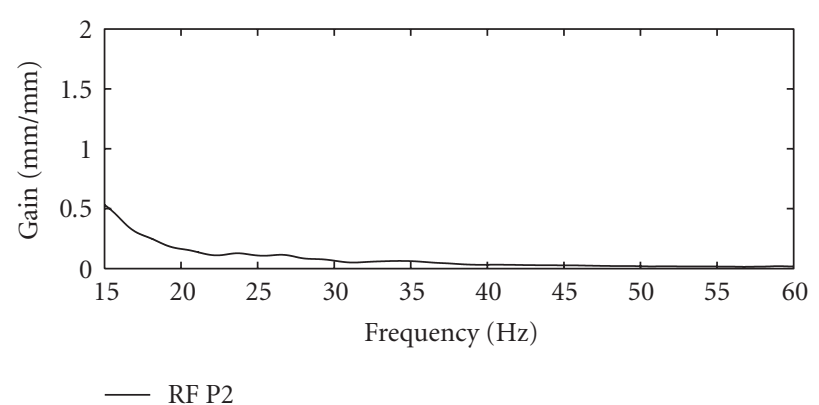

(b)

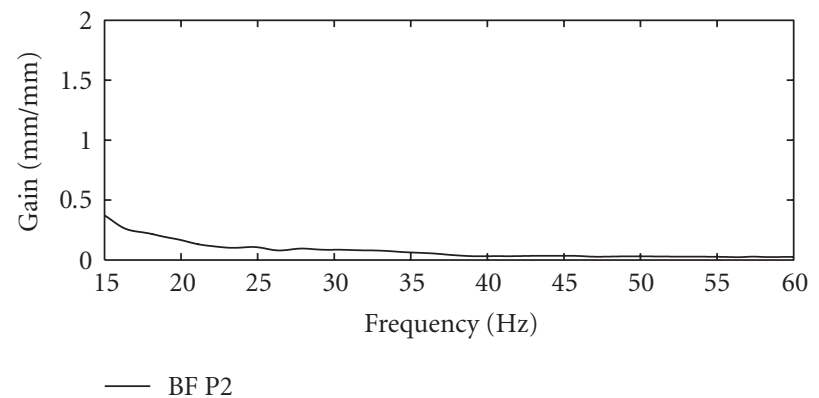

(d)

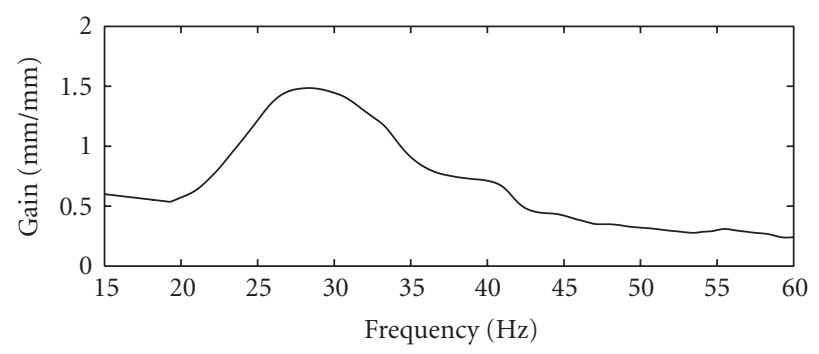

- GLP2

(f)

FIGURE 9: An example of estimated normalised resultant muscle displacements over frequency with respect to position P1 ((a) for Rectus Femoris, (c) for Biceps Femoris, (e) for Gastrocnemius Lateralis) and P2 ((b) for Rectus Femoris, (d) for Biceps Femoris, (f) for Gastrocnemius Lateralis).

TABLE 1: Frequency response parameters: mean \pm SD.

\begin{tabular}{lcccc}
\hline & Fr $(\mathrm{Hz})$ & $\mathrm{Mr}(\mathrm{mm} / \mathrm{mm})$ & $\mathrm{Q}$ & $R_{1}^{2}$ \\
\hline RF P1 & $20.50 \pm 1.84$ & $1.42 \pm 0.37$ & $3.49 \pm 1.24$ & $0.95 \pm 0.05$ \\
BF P1 & $19.90 \pm 2.44$ & $1.12 \pm 0.18$ & $2.56 \pm 1.06$ & $0.95 \pm 0.06$ \\
GL P2 & $30.30 \pm 8.27$ & $1.04 \pm 0.36$ & $2.49 \pm 1.02$ & $0.89 \pm 0.11$ \\
\hline
\end{tabular}

low frequencies $(0$ to $5 \mathrm{~Hz}$ ) [31], while frequency content of a muscle motion induced by vibration basically consists of the fundamental vibration frequency (i.e., from $15-60 \mathrm{~Hz}$ in these trials) and its superior harmonics.

Therefore, in order to consider only vibration related muscle oscillations, standard high pass filtering was combined with double integration providing reliable estimation of the actual muscle displacements.

Three-dimensional muscle motion resulted relatively complex: each of the motion component (along the relative $x-, y-, z$-axes) resulted not always purely sinusoidal neither in phase to each other.

$\mathrm{RF}$ and $\mathrm{BF}$ resonance were observed below $25 \mathrm{~Hz}$ while individuals maintain position $\mathrm{P} 1$; similarly a GL resonant response was found above $30 \mathrm{~Hz}$ in position $\mathrm{P} 2$.

The analysis of the resultant estimated displacements confirmed that BF and RF muscles achieved the maximum oscillation (i.e., receive the maximal vibration stimulation) in position P1 while GL maximal oscillation was achieved in position P2. 


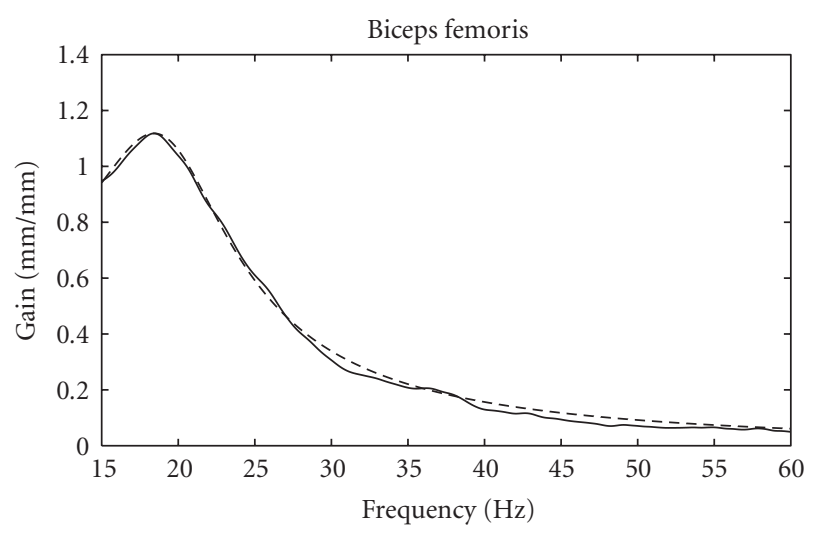

Figure 10: An example of second-order system data fitting: continuous line represent the estimated muscle transfer function while the dotted line shows the second-order approximation.

TABLE 2: Contra-lateral analysis: mean \pm SD.

\begin{tabular}{lcc}
\hline & $\Delta$ Fr $(\mathrm{Hz})$ & $R_{2}^{2}$ \\
\hline RF P1 & $0.26 \pm 1.85$ & $0.95 \pm 0.04$ \\
BF P1 & $0.39 \pm 2.79$ & $0.95 \pm 0.03$ \\
GL P1 & $X$ & $0.94 \pm 0.04$ \\
RF P2 & $X$ & $0.88 \pm 0.09$ \\
BF P2 & $X$ & $0.94 \pm 0.06$ \\
\hline
\end{tabular}

It is worth mentioning that only for this posture/muscle combinations a resonant-like behaviour was clearly visible; however further investigations will be considered to assess the variability of muscle response after multiple vibratory stimulations and different rest periods.

Since experimental data resembled a second-order system like response (suggesting a kinematic equivalent behaviour of a wobbling damped oscillating mass) a simple data fitting was performed. The high correlation coefficient $\left(R_{1}^{2}\right.$, see Table 1) between the empirical frequency response and analytical second-order transfer function then confirmed the validity of the assumption.

Contra-lateral analysis showed nonsignificant differences between dominant and nondominant lower limb; both lower limbs muscles motion resulted to be synchronous and symmetric with respect to the sagittal-plane.

However muscles mechanical resonance characteristics (frequency and dumping factor) depended on subject.

In conclusion, as highlighted in this study, vibratory induced muscle oscillations strongly depend on subject's posture; as suggested, our results confirmed the meaning of a proper positioning in vibratory stimulus transmission.

Since WBV training is included in many fitness and rehabilitation centres exercise programmes, improvements in current knowledge would be helpful in discovering effective exercise protocols.

Assuming the hypothesis of correlation between muscle motion and EMG activity [25], WBV muscle stimulation programmes have to take into account the posture hold by subject on platform, and in turn, the local muscle mechanical oscillation (i.e., local muscle stimulation). In particular our results suggested that $\mathrm{BF}$ and $\mathrm{RF}$ muscles may receive the maximal stimulus in position P1 while the for GL muscle stimulation may be preferable position $\mathrm{P} 2$.

However, maximizing muscles motion could act in producing more muscle lengthening, and in turn higher muscle activation. In addition, since this study involved untrained male subjects, studies with female subjects should be performed to compare with the results obtained for male subjects.

\section{Acknowledgment}

The authors are grateful to TSEM S.p.A. for providing vibrating platform device.

\section{References}

[1] J. R. Burke, M. C. Schutten, D. M. Koceja, and G. Kamen, "Age-dependent effects of muscle vibration and the Jendrassik maneuver on the patellar tendon reflex response," Archives of Physical Medicine and Rehabilitation, vol. 77, no. 6, pp. 600604, 1996.

[2] M. Cardinale and J. Lim, "Electromyography activity of vastus lateralis muscle during whole-body vibrations of different frequencies," Journal of Strength and Conditioning Research, vol. 17, no. 3, pp. 621-624, 2003.

[3] M. Cardinale and J. Wakeling, "Whole body vibration exercise: are vibrations good for you?" British Journal of Sports Medicine, vol. 39, no. 9, pp. 585-589, 2005.

[4] C. Delecluse, M. Roelants, and S. Verschueren, "Strength increase after whole-body vibration compared with resistance training," Medicine and Science in Sports and Exercise, vol. 35, no. 6, pp. 1033-1041, 2003.

[5] J. E. Desmedt and E. Godaux, "Vibration-induced discharge patterns of single motor units in the masseter muscle in man," Journal of Physiology, vol. 253, no. 2, pp. 429-442, 1975.

[6] C. Bosco, R. Colli, E. Introini, et al., "Adaptive responses of human skeletal muscle to vibration exposure," Clinical Physiology, vol. 19, no. 2, pp. 183-187, 1999.

[7] C. Bosco, M. Cardinale, and O. Tsarpela, "Influence of vibration on mechanical power and electromyogram activity in human arm flexor muscles," European Journal of Applied Physiology and Occupational Physiology, vol. 79, no. 4, pp. 306311, 1999.

[8] I. Bautmans, E. Van Hees, J.-C. Lemper, and T. Mets, "The feasibility of whole body vibration in institutionalised elderly persons and its influence on muscle performance, balance and mobility: a randomised controlled trial," BMC Geriatrics, vol. 5 , article 17, p. 8, 2005.

[9] C. Bosco, M. Iacovelli, O. Tsarpela, et al., "Hormonal responses to whole-body vibration in men," European Journal of Applied Physiology, vol. 81, no. 6, pp. 449-454, 2000.

[10] C. Bosco, M. Cardinale, O. Tsarpela, et al., "The influence of whole body vibration on jumping performance," Biology of Sport, vol. 15, no. 3, pp. 157-164, 1998.

[11] J. A. Eisman, "Good, good, good... good vibrations: the best option for better bones?" The Lancet, vol. 358, no. 9297, pp. 1924-1925, 2001.

[12] I. Homma, T. Nagai, T. Sakai, M. Ohashi, M. Beppu, and K. Yonemoto, "Effect of chest wall vibration on ventilation in patients with spinal cord lesion," Journal of Applied Physiology, vol. 50, no. 1, pp. 107-111, 1981. 
[13] V. B. Issurin, D. G. Liebermann, and G. Tenenbaum, "Effect of vibratory stimulation training on maximal force and flexibility," Journal of Sports Sciences, vol. 12, no. 6, pp. 561566, 1994.

[14] K. Kerschan-Schindl, S. Grampp, C. Henk, et al., "Wholebody vibration exercise leads to alterations in muscle blood volume," Clinical Physiology, vol. 21, no. 3, pp. 377-382, 2001.

[15] S. Verschueren, M. Roelants, C. Delecluse, S. Swinnen, D. Vanderschueren, and S. Boonen, "Effect of 6-month whole body vibration training on hip density, muscle strength, and postural control in postmenopausal women: a randomized controlled pilot study," Journal of Bone and Mineral Research, vol. 19, no. 3, pp. 352-359, 2004.

[16] C. D. Marsden, J. C. Meadows, and H. J. Hodgson, "Observations on the reflex response to muscle vibration in man and its voluntary control,” Brain, vol. 92, no. 4, pp. 829-846, 1969.

[17] J. Mester, P. Spitzenfeil, J. Schwarzer, and F. Seifriz, "Biological reaction to vibration-Implications for sport," Journal of Science and Medicine in Sport, vol. 2, no. 3, pp. 211-226, 1999.

[18] J. Mester, H. Kleinoder, and Z. Yue, "Vibration training: benefits and risks," Journal of Biomechanics, vol. 39, no. 6, pp. 1056-1065, 2006.

[19] J. Rittweger, H. Schiessl, and D. Felsenberg, "Oxygen uptake during whole-body vibration exercise: comparison with squatting as a slow voluntary movement," European Journal of Applied Physiology, vol. 86, no. 2, pp. 169-173, 2001.

[20] C. T. Rubin, R. Recker, D. Cullen, J. Ryaby, J. McCabe, and K. McLeod, "Prevention of postmenopausal bone loss by a lowmagnitude, high-frequency mechanical stimuli: a clinical trial assessing compliance, efficacy, and safety," Journal of Bone and Mineral Research, vol. 19, no. 3, pp. 343-351, 2004.

[21] S. Torvinen, P. Kannus, H. Sievänen, et al., "Effect of a vibration exposure on muscular performance and body balance. Randomized cross-over study," Clinical Physiology and Functional Imaging, vol. 22, no. 2, pp. 145-152, 2002.

[22] C. Bosco, "Automatic device for optimized muscular stimulation,” US patent no. WO01/56650, January 2001.

[23] A. Fratini, M. Cesarelli, P. Bifulco, and M. Romano, "Relevance of motion artifact in electromyography recordings during vibration treatment," Journal of Electromyography and Kinesiology, vol. 19, no. 4, pp. 710-718, 2009.

[24] H. De Talhouet and J. G. Webster, "The origin of skin-stretchcaused motion artifacts under electrodes," Physiological Measurement, vol. 17, no. 2, pp. 81-93, 1996.

[25] A. Fratini, P. Bifulco, M. Cesarelli, G. Pasquariello, M. Romano, and A. La Gatta, "Correspondence between muscle motion and EMG activity during whole body vibration," in Proceedings of the 4th European Conference of the International Federation for Medical and Biological Engineering (IFMBE'08), vol. 22, pp. 2069-2072, Antwerp, Belgium, 2008.

[26] J. M. Wakeling and A.-M. Liphardt, "Task-specific recruitment of motor units for vibration damping," Journal of Biomechanics, vol. 39, no. 7, pp. 1342-1346, 2006.

[27] J. M. Wakeling, B. M. Nigg, and A. I. Rozitis, "Muscle activity damps the soft tissue resonance that occurs in response to pulsed and continuous vibrations," Journal of Applied Physiology, vol. 93, no. 3, pp. 1093-1103, 2002.

[28] J. E. Desmedt and E. Godaux, "Mechanism of the vibration paradox: excitatory and inhibitory effects of tendon vibration on single soleus muscle motor units in man," Journal of Physiology, vol. 285, pp. 197-207, 1978.

[29] B. Harazin and J. Grzesik, "The transmission of vertical wholebody vibration to the body segments of standing subjects,"
Journal of Sound and Vibration, vol. 215, no. 4, pp. 775-787, 1998.

[30] D. A. Nowak, K. Rosenkranz, J. Hermsdörfer, and J. Rothwell, "Memory for fingertip forces: passive hand muscle vibration interferes with predictive grip force scaling," Experimental Brain Research, vol. 156, no. 4, pp. 444-450, 2004.

[31] T. E. Prieto, J. B. Myklebust, R. G. Hoffmann, E. G. Lovett, and B. M. Myklebust, "Measures of postural steadiness: differences between healthy young and elderly adults," IEEE Transactions on Biomedical Engineering, vol. 43, no. 9, pp. 956-966, 1996. 